\title{
Infections in asthma and allergy
}

\author{
S Lewis
}

The role of infection in asthma is presently a paradox. While respiratory syncytial virus, among others, has long been believed to instigate immunological changes which lead to asthma, and rhinovirus might be an important contributory factor in the exacerbation of asthma symptoms in children and adults, there is increasing speculation that infections in early life have a protective role in the development of allergy and asthma. The motivation for this theory was the observation of a strong relationship between birth order and the risk of allergic disease and generic atopy which has consistently emerged from British cohorts spanning the last 40 years. ${ }^{1-3}$ One of the appeals of the infection hypothesis is its undoubted capacity to explain the increase in allergic disease in developed societies over recent years as an inevitable consequence of improved hygiene standards, increased immunisation, and reduced contact between children owing to declining family size and less crowded homes. A theory which was once based exclusively on epidemiological associations has now gained support on immunological grounds. As the mechanisms which control the immune response to allergen and the course of the events in early life leading to maturation of this system become better understood, it is evident that the microbial environment of early life is a potential influence on this process. ${ }^{4}$ Neither epidemiology nor immunology yet provide much evidence on the nature of the infection or infections likely to be involved, but each supplies some useful, though sometimes disparate, clues.

In this issue of Thorax Farooqi and Hopkin have taken a retrospective look at all the available health records of a relatively recent (1975-1984) birth group taken from a single general practice in Oxfordshire, and examined links between a diversity of early childhood infections and the subsequent diagnosis of allergic disease. ${ }^{5}$ This historical cohort study is relatively small, comprising just under 2000 subjects, and the resultant lack of power may explain the failure of the authors to detect an association between birth order and doctor diagnosed asthma, eczema, or hay fever. Nevertheless, a study of this size had a very good chance (at least $80 \%$ probability) of detecting a halving of risk of allergic disease in children with multiple older siblings such as that previously described. It is possible that the relationship between birth order and allergy has weakened over time, ${ }^{1}$ perhaps as exposure to the underlying influential factor-whether infection or otherwise - has become more ubiquitous. Alternatively, the disparity may reflect biases introduced in the study by the influence that parental consulting behaviour is likely to have on the clinical diagnosis of allergic disease. The birth order effect would tend to be reduced, for example, if the same symptoms were presented earlier in a child with an affected older sibling. It is this potential for consulting and diagnostic behaviour to affect both the allergic outcome and the determination of infection exposure which is the primary source of bias and confounding in this study. Nevertheless, by using general practice records these authors are able to look at a wider range of early childhood infections and immunisations than has previously been considered and to explore the temporality of the exposure-response relationship.

From an epidemiological standpoint, several of the common infectious diseases of childhood are prime candidates for investigation. The proportion of children contracting whooping cough, measles, and mumps has reduced substantially in the developed world as a result of the introduction of vaccine, which happened prior to the earliest reports of increases in the prevalence of allergic disease for all but mumps. Acquisition of measles infection and the age and severity of infection are strongly dependent upon familial factors, especially crowding in the home ${ }^{6}$; the same is likely to be true of the other childhood infectious diseases. The findings reported by Farooqi and Hopkin are remarkably consistent with a similar study published earlier this year in Thorax ${ }^{8}$ in showing no effect, or even a positive association, between asthma, eczema, and hay fever and each of the childhood infectious diseases except measles. It is notable that rates of chickenpox, which have changed very little over recent years, are substantially lower in this new study than in the previous one which was based on parental responses, suggesting that common childhood infectious diseases are frequently not reported to, or else recorded by, the GP. It is therefore possible that parental consulting habits confound the positive associations reported by Farooqi and Hopkin and none were significant in multivariate analysis with adjustment for frequency of GP consultation. On the other hand, in both recent studies the association with measles is opposite to that seen for all of the other infections such that measles has an apparently protective effect on one or more of asthma, eczema, and hay fever which was too small to reach statistical significance in either study. Measles was first implicated in data from West Africa where children with measles infection were significantly less likely to be atopic, assessed by allergen skin sensitivity, than those receiving measles immunisation. ${ }^{6}$ The relevance of measles is intriguing therefore, but overall these studies provide little support for a role for any of the other common childhood infectious diseases addressed in this study.

One possibility raised by the West African data is that, rather than measles infection having some protective role against the development of allergy, measles immunisation might promote its development. The immune response induced by measles ${ }^{9}$ and other vaccines or their adjuvants is consistent with the possibility that increased availability of vaccination rather than reduced exposure to infection might have contributed to the increased prevalence of allergic disease. This question is difficult to address in most developed societies where most vaccinations are now too ubiquitous and the few who remain unvaccinated are an atypical group. ${ }^{10}$ Farooqi and Hopkin have looked specifically at two vaccinations introduced relatively recently, pertussis and measles, and found that most of the subjects received both vaccines, but that doctor diagnosed asthma, eczema, and hay fever were increased in those who received pertussis immunisation compared with the $27 \%$ who did not. Pertussis vaccine has been previously linked to asthma ${ }^{11}$ and its adjuvants are known to stimulate $\operatorname{IgE}$ production. ${ }^{12}$ Nevertheless, if pertussis vaccination does alter the risk of allergic disease it is unlikely to explain its changing prevalence, for the temporal pattern in the uptake of pertussis vaccination (high in the early 1970s, dropping sharply to $30 \%$ nationally by 1975 following public fears about the safety and efficacy of the vaccine, and rising rapidly for the remainder of the 1970s and 1980s) is inconsistent with the steady increase in the prevalence of 
asthma in successive birth cohorts during the same period. ${ }^{13}$ Since the same alum adjuvant is also present in diphtheria and tetanus vaccines (introduced during the 1940 s and 1960 s, respectively), it is still possible that the positive association with pertussis vaccination reflects the involvement of other vaccinations. Overall, however, the vaccination argument does not fit as intuitively with the strong birth order effect in allergic disease as does infection.

Infections transmitted easily between children, such as those operating by the oral-oral or faecal-oral route, would be most in keeping with the birth order effect. Interest has focused on respiratory infections because, epidemiologically, exposure bears a strong relationship with family size ${ }^{14}$ and, immunologically, this places the $T$ cell response to infection at the site of contact with inhaled allergen. ${ }^{15}$ In one of the first studies to provide direct evidence relating infection to allergy Martinez et al found persistently low IgE levels in children experiencing a non-wheezing lower respiratory tract infection in the first few years of life. ${ }^{16}$ Yet, despite removing from analysis all those who wheezed during a lower respiratory tract infection, Farooqi and Hopkin found the diagnosis of allergic disease to be positively rather than negatively associated with all types of infection of the respiratory tract. This data set was too small and the potential biases too great to unravel the hypothetically diametrically opposed effects of respiratory viral infection on the inception of asthma and on the development of atopy. Only one study to date has suggested that infections transmitted by the faecal-oral route might be important ${ }^{17}$; exposure to hepatitis A was inversely associated with atopy but did not explain the birth order effect. One interpretation is that, if infection modifies the risk of atopy, more than a single exposure to a specific infection is involved; this is more consistent, perhaps, with the immunological picture.

The allergen specific $\mathrm{T}$ cell response, which in the mature immune system is polarised towards the Th2 cytokine profile in atopic subjects but the Th1 profile in non-atopic subjects, is unstable during early life, perhaps reaching an equilibrium only after the first $4-5$ years. The balance attained may be determined by the cytokine environment, either at the first or each of multiple exposures to a specific antigen, and influenced therefore by the Th1 cytokine response to a succession of bacterial and viral pathogens. ${ }^{18}$ The more important part of the development process, however, may be the length of time for this balance to accrue, such that those destined to become atopic take longer to achieve maturation of immune competence. ${ }^{19}$ The environmental drive for maturation may be confrontation with the microbial environment especially, perhaps, of the gut. ${ }^{4}$ In animal models normal immune development is inhibited whilst the stomach is a pathogen free environment ${ }^{20}$; a similar condition is created in humans in response to antibiotics. By this argument antibiotic use in early childhood might be hypothesised to increase the subsequent risk of atopy and the findings of Farooqi and Hopkins are consistent with this hypothesis, though bias arising from the prescription of antibiotics for symptoms marking the onset of allergic disease is an alternative and likely explanation. If this were the case, we would expect the relationship with allergic disease to be stronger in relation to the use of antibiotics for respiratory tract infection than for urinary or other non-respiratory infection. On balance the opposite is true in the group studied by Farooqi and Hopkin, though the confidence intervals are wide and overlapping. A causal link between antibiotic use and allergy is also supported by the strength and dose-response pattern of the association in this study and the specificity of the effect to antibiotic use in the early years. Nevertheless, antibiotic use would seem to have little connection with birth order and thus, if the effect is real, this puzzle remains unsolved.

We have therefore advanced over very recent years from an infection hypothesis based on indirect epidemiological observations to a position where immunology can provide at least two potential mechanisms, but the direct evidence is still inconclusive. Interestingly, if antibiotics are involved, we may need to modify the original hypothesis to encompass all aspects of the microbial environment, commensals as well as pathogens. There is clearly much here worthy of further research. On the evidence of the data reported by Farooqi and Hopkin, more epidemiological studies in younger cohorts of developed countries may not be helpful because even some of what we once consider common childhood infections such as measles now occur in less than $5 \%$ of the population, whilst most children are prescribed antibiotics at some time in early childhood. We may therefore have to rely on studies of older populations, despite all the associated problems and biases involved in retrospectively assessing exposures in early childhood. Ultimately, however, the most convincing answers seem likely to come from similar studies in the developing world.

Respiratory Medicine Unit,

$S$ A LEWIS

City Hospital,

Nottingham NG5 $1 P B$

UK

1 Butland BK, Strachan DP, Lewis S, et al. Investigation into the increase in hay fever and eczema at age 16 observed between the 1958 and 1970 British birth cohorts. BMF 1997;315:717-21.

2 Strachan DP, Griffiths JM, Anderson HR, et al. Allergic sensitisation and position in the sibship: a national study of young British adults. Thorax 1994;49:1053P.

3 Strachan DP, Taylor EM, Carpenter RG. Family structure, neonatal infection, and hay fever in adolescence. Arch Dis Child 1996;74:422-6.

4 Holt PG, Sly PD, Bjorksten B. Atopic versus infectious diseases in childhood: a question of balance? Pediatr Allergy Immunol 1997;8:53-8.

5 Farooqi IS, Hopkin J. Early childhood infection and atopic disorder. Thorax 1998;53:927-32.

6 Shaheen SO, Aaby P, Hall AJ, et al. Measles and atopy in Guinea-Bissau. Lancet 1996;347:1792-6.

7 Aaby P, Bukh J, Lisse IM, et al. Overcrowding and intensive exposure as determinants of measles mortality. Am f Epidemiol 1998;120:49-59.

8 Bodner C, Godden D, Seaton A. Family size, childhood infections and atopic disease. Thorax 1998;53:28-32.

9 Ward BJ, Griffin DE. Changes in cytokine production after measles virus vaccination: predominant production of IL-4 suggests induction of a Th2 response. Clin Immunol Immunopathol 1993;67:171-7.

$10 \mathrm{Kemp} T$, Pearce N, Fitzharris $\mathrm{P}$, et al. Is infant immunization a risk factor for childhood asthma or allergy? Epidemiology 1997;8:678-80.

11 Odent MR, Culpin EE, Khnmel T. Pertussis vaccination and asthma: is there a link? $\mathcal{F} A M A$ 1994;272:592-3.

12 Pauwels R, Van der Straeten M, Platteau M, et al. The non-specific enhancement of allergy. 1. In vivo effects of Bordetella pertussis vaccine on IgE synthesis. Allergy 1983;38:239-46.

13 Burney PGJ, Chinn S, Rona RJ. Has the prevalence of asthma increased in children? Evidence from the national study of health and growth 1973-86. BMF 1990;300:1306-10

14 Gardner G, Frank AL, Taber LH . Effects of social and family factors on viral respiratory infection and illness in the first year of life. $\mathcal{F}$ Epidemiol Community Health 1984;38:42-8.

15 Holt PG. Environmental factors and primary T-cell sensitisation to inhalant allergens: reappraisal of the role of infections and air pollution. Pediatr Allergy Immunol 1995;6:1-10.

16 Martinez FD, Stern DA, Wright AL, et al. Association of non-wheezing lower respiratory tract illnesses in early life with persistently diminished serum IgE levels. Thorax 1995;50:1067-72.

17 Matricardi PM, Rosmini F, Ferrigno L, et al. Cross sectional retrospective study of prevalence of atopy among Italian military students with antibodies against hepatitis A virus. BMf 1997;314:999-1003.

18 Romagnani S. Human TH1 and TH2 subsets: regulation of differentiation and role in protection and immunopathology. Int Arch Allergy Immunol 1992;98:279-85.

19 Holt PG, Clough J, Holt B. Genetic risk for atopy is associated with delayed maturation of T cell competence. Clin Exp Allergy 1992;22:1093-9.

20 Inagaki $\mathrm{H}$, Suzuki $\mathrm{T}$, Nomoto $\mathrm{K}$, et al. Increased susceptibility to primary infection with Listeria monocytogenes in germfree mice may be due to lack of accumulation of L-selectin $+\mathrm{CD} 44++\mathrm{T}$ cells in sites of inflammation. Infect Immun 1996;64:3280-7. 